\title{
Analysis of Unsteady Axisymmetric Squeezing Fluid Flow with Slip and No-Slip Boundaries Using OHAM
}

\author{
Mubashir Qayyum, Hamid Khan, M. Tariq Rahim, and Inayat Ullah \\ Department of Mathematics, National University of Computer \& Emerging Sciences, FAST Peshawar Campus, \\ Peshawar 25000, Pakistan \\ Correspondence should be addressed to Hamid Khan; hamid.khan@nu.edu.pk
}

Received 27 May 2014; Revised 11 August 2014; Accepted 12 September 2014

Academic Editor: María Isabel Herreros

Copyright (C) 2015 Mubashir Qayyum et al. This is an open access article distributed under the Creative Commons Attribution License, which permits unrestricted use, distribution, and reproduction in any medium, provided the original work is properly cited.

In this manuscript, An unsteady axisymmetric flow of nonconducting, Newtonian fluid squeezed between two circular plates is studied with slip and no-slip boundaries. Using similarity transformation, the system of nonlinear partial differential equations is reduced to a single fourth order ordinary differential equation. The resulting boundary value problems are solved by optimal homotopy asymptotic method (OHAM) and fourth order explicit Runge-Kutta method (RK4). It is observed that the results obtained from OHAM are in good agreement with numerical results by means of residuals. Furthermore, the effects of various dimensionless parameters on the velocity profiles are investigated graphically.

\section{Introduction}

The squeezing of an incompressible viscous fluid between two parallel plates is an essential type of flow that is frequently observed in many hydro dynamical tools and machines. In food industries, squeezing flows have several applications particularly in chemical engineering $[1,2]$. Compression and injection molding, polymer processing, and modeling of lubrication system are some practical examples of squeezing flows. The modeling and analysis of squeezing flow has started in nineteenth century and continues to receive significant attention due to its vast applications in biophysical and physical sciences. The initial work in squeezing flows has been done by Stefan [3], who developed an ad hoc asymptotic solution of Newtonian fluids. An explicit solution of the squeeze flow, considering inertial terms, has been established by Thorpe and Shaw [4]. However, P. S. Gupta and A. S. Gupta [5] proved that the solution set up by [4] fails to satisfy the boundary conditions. Considering fluid inertia effects, Elkouh [6] studied the squeeze film between two plane annuli. Verma [7] and Singh et al. [8] have conducted Numerical solutions of the squeezing flow between parallel plates. Leider and Bird [9] performed theoretical analysis for squeezing flow of power-law fluid between parallel disks.
Analytic solution for the squeezing flow of viscous fluid between two parallel disks with suction or blowing effect has been proposed by Domairry and Aziz [10]. Islam et al. [11] studied Newtonian squeezing fluid flow in a porous medium channel. Ullah et al. [12] discussed the Newtonian fluid flow with slip boundary condition keeping MHD effect into account. Siddiqui et al. [13] investigated the unsteady squeezing flow of viscous fluid with magnetic field. Apart from the mentioned researchers, other prominent scholars have conducted various theoretical and experimental studies of squeezing flows [14-17].

The difference between fluid and boundary velocity is proportional to the shear stress at the boundary. The dimension of proportionality constant is length, which is known as slip parameter. In fluids with elastic character, slip condition has great importance [18]. It has many applications in medical sciences, for instance, polishing artificial heart valves [19]. There are various situations in which no-slip boundary condition is inappropriate. Some of these situations include polymeric liquids when the weight of molecule is high, flow on multiple interfaces, fluids containing concerted suspensions, and thin film problems. The general boundary condition which shows the fluid slip at the wall was initially proposed by Navier [20]. Recently, Ebaid [21] studied 
the effect of magnetic field in Newtonian fluid in an asymmetric channel with wall slip conditions.

Most of scientific incidents are modeled by nonlinear partial or ordinary differential equations. In literature, we have variety of perturbation techniques which can solve nonlinear boundary value problems analytically. But the limitations of these techniques are based on the assumption of small parameters. Detailed review of these methods is given by $\mathrm{He}$ [22]. In recent times, the ideas of Homotopy and Perturbation have been combined together. Liao [23] and $\mathrm{He}$ $[24,25]$ have done the primary work in this regard. In series of papers, Marinca with various scholars used OHAM to find the approximate solution of nonlinear differential equations arising in heat transfer, steady flow of a fourth-grade fluid, and thin film flow [26-28].

In this work, OHAM is used to analyze an unsteady squeezing fluid flow between two circular nonrotating disks with slip and no-slip boundary conditions. In addition, movement of the circular plates is considered to be symmetric about the axial line and the fluid is considered to be Newtonian, incompressible and viscous. Sections 2 and 3 include the description and mathematical formulation of the problem. Sections 4, 5, and 6 present the basic theory of OHAM and its application in case of no-slip and slip boundaries. Results and discussions are given in Section 7 while conclusions are mentioned in Section 8.

\section{Description of the Problem}

The unsteady axisymmetric squeezing flow of incompressible Newtonian fluid with density $\rho$, viscosity $\mu$ and kinematic viscosity $v$, squeezed between two circular plates having speed $v_{w}(t)$ is considered. It is assumed that at any time $t$, the distance between two circular plates is $2 s(t)$. It is also assumed that $r$-axis is the central axis of the channel while $z$-axis is taken normal to it. Plates move symmetrically with respect to the central axis $z=0$ while the flow is axisymmetric about $r=0$. The longitudinal and normal velocity components in radial and axial directions are $u_{r}(r, z, t)$ and $u_{z}(r, z, t)$, respectively. The geometrical interpretation of the problem is given in Figure 1.

\section{Mathematical Formulation}

The governing equations of motion are

$$
\begin{gathered}
\nabla \cdot \mathbf{U}=0, \\
\rho\left[\frac{\partial \mathbf{U}}{\partial t}+(\mathbf{U} \cdot \nabla) \mathbf{U}\right]=\rho f+\nabla \cdot \mathbf{T}
\end{gathered}
$$

where

$$
\begin{gathered}
\mathbf{T}=-p \mathbf{I}+\mu \mathbf{A}_{1}, \\
\mathbf{A}_{1}=\nabla \mathbf{U}+(\nabla \mathbf{U})^{t},
\end{gathered}
$$

and $\mathbf{U}$ is the velocity vector, $p$ is the pressure, $f$ is the body force, $\mathbf{T}$ is the Cauchy stress tensor, $\mathbf{A}_{1}$ is the Rivlin-Ericksen

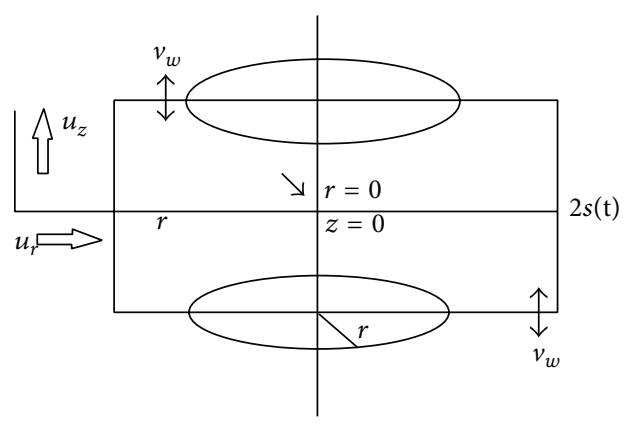

FIGURE 1: Geometrical interpretation of the problem.

tensor, and $\mu$ is the coefficient of viscosity. Now we formulate the unsteady two-dimensional flow. Let us assume that

$$
\mathbf{U}=\left[u_{r}(r, z, t), 0, u_{z}(r, z, t)\right],
$$

and introduce the vorticity function $\Omega(r, z, t)$ and generalized pressure $h(r, z, t)$ as

$$
\begin{gathered}
\Omega(r, z, t)=\frac{\partial u_{z}}{\partial r}-\frac{\partial u_{r}}{\partial z}, \\
h(r, z, t)=\frac{\rho}{2}\left[u_{r}^{2}+u_{z}^{2}\right]+p .
\end{gathered}
$$

Equations (1) are reduced to

$$
\begin{gathered}
\frac{\partial u_{r}}{\partial r}+\frac{u_{r}}{r}+\frac{\partial u_{z}}{\partial z}=0 \\
\frac{\partial h}{\partial r}+\rho\left(\frac{\partial u_{r}}{\partial t}-u_{z} \Omega\right)=-\mu \frac{\partial \Omega}{\partial z} \\
\frac{\partial h}{\partial z}+\rho\left(\frac{\partial u_{z}}{\partial t}+u_{r} \Omega\right)=\frac{\mu}{r} \frac{\partial}{\partial r}(r \Omega) .
\end{gathered}
$$

The boundary conditions on $u_{r}(r, z, t)$ and $u_{z}(r, z, t)$ are

$$
\begin{gathered}
u_{r}(r, z, t)=0, \quad u_{z}(r, z, t)=v_{w}(t) \quad \text { at } z=s \\
\frac{\partial}{\partial z} u_{r}(r, z, t)=0, \quad u_{z}(r, z, t)=0 \quad \text { at } z=0,
\end{gathered}
$$

where $v_{w}(t)=d s / d t$ is the velocity of the plates. The boundary conditions (7) are due to symmetry at $z=0$ and no-slip at the upper plate when $z=s$. If we introduce the dimensionless parameter

$$
\eta=\frac{z}{s(t)} .
$$

Equations (4) and (6) transforms to

$$
\begin{gathered}
\Omega(r, z, t)=\frac{\partial u_{z}}{\partial r}-\frac{1}{s} \frac{\partial u_{r}}{\partial \eta} \\
\frac{\partial u_{r}}{\partial r}+\frac{u_{r}}{r}+\frac{1}{s} \frac{\partial u_{z}}{\partial \eta}=0 \\
\frac{\partial h}{\partial r}+\rho\left(\frac{\partial u_{r}}{\partial t}-u_{z} \Omega\right)=-\frac{\mu}{s} \frac{\partial \Omega}{\partial \eta} \\
\frac{1}{s} \frac{\partial h}{\partial \eta}+\rho\left(\frac{\partial u_{z}}{\partial t}+u_{r} \Omega\right)=\frac{\mu}{r} \frac{\partial}{\partial r}(r \Omega) .
\end{gathered}
$$


The boundary conditions on $u_{r}$ and $u_{z}$ are

$$
\begin{gathered}
u_{r}=0, \quad u_{z}=v_{w}(t) \quad \text { at } \eta=1 \\
\frac{\partial u_{r}}{\partial \eta}=0, \quad u_{z}=0 \quad \text { at } \eta=0 .
\end{gathered}
$$

After eliminating the generalized pressure between (11) and (12), we obtained

$$
\rho\left[\frac{\partial \Omega}{\partial t}+u_{r} \frac{\partial \Omega}{\partial r}+\frac{u_{z}}{s} \frac{\partial \Omega}{\partial \eta}-\frac{u_{r}}{r} \Omega\right]=\mu\left[\nabla^{2} \Omega-\frac{\Omega}{r^{2}}\right],
$$

where $\nabla^{2}$ is the Laplacian operator.

Defining velocity components as [5]

$$
\begin{gathered}
u_{r}=-\frac{r}{2 s(t)} v_{w}(t) g^{\prime}(\eta), \\
u_{z}=v_{w}(t) g(\eta)
\end{gathered}
$$

we see that (10) is identically satisfied and (14) becomes

$$
\frac{d^{4} g}{d \eta^{4}}+R\left[(\eta-g) \frac{d^{3} g}{d \eta^{3}}+2 \frac{d^{2} g}{d \eta^{2}}\right]-Q \frac{d^{2} g}{d \eta^{2}}=0
$$

where

$$
R=\frac{s v_{w}}{v}, \quad Q=\frac{s^{2}}{v v_{w}} \frac{d v_{w}}{d t} .
$$

Here both $R$ and $Q$ are functions of $t$ but we consider $R$ and $Q$ constants for similarity solution. Since $v_{w}=d s / d t$, Integrate first equation of (17), we get

$$
s(t)=(W t+S)^{1 / 2}
$$

where $W$ and $S$ are constants. The plates move away from each other symmetrically with respect to $\eta$ when $W>0$ and $S>$ 0 . Also the plates approach to each other and squeezing flow exists with similar velocity profiles when $W<0, S>0$, and $s(t)>0$. From (17) and (18) it follows that $Q=-R$. Then (16) becomes

$$
\frac{d^{4} g}{d \eta^{4}}+R\left[(\eta-g) \frac{d^{3} g}{d \eta^{3}}+3 \frac{d^{2} g}{d \eta^{2}}\right]=0 .
$$

Using (13) and (15) we determine the boundary conditions in case of no-slip and slip at the upper plate as follows:

$$
\begin{aligned}
& \begin{array}{ll}
g(1)=1, & g^{\prime}(1)=0 \\
g(0)=0, & g^{\prime \prime}(0)=0
\end{array} \quad \text { (No-slip at the wall) }
\end{aligned}
$$

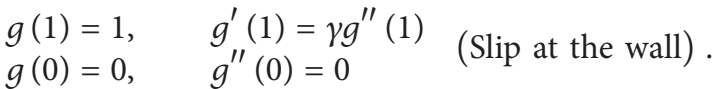

\section{Basic Theory of OHAM [26, 29-32]}

Let us apply OHAM to the following differential equation:

$$
\mathfrak{\Im}[v(x)]+f(x)+\aleph[v(x)]=0, \quad B\left(v, \frac{d v}{d x}\right)=0,
$$

where $x$ represents an independent variable, $v(x)$ is unknown function and $f(x)$ is known function. $B, \aleph, \mathfrak{\Im}$ are boundary, nonlinear, and linear operators, respectively.

According to OHAM, we construct Homotopy $\Phi(x, p)$ : $\mathbb{R} \times[0,1] \rightarrow \mathbb{R}$ which satisfies

$$
\begin{gathered}
(1-p)[\mathfrak{I}(\Phi(x, p))+f(x)] \\
=h(p)[\Im(\Phi(x, p))+f(x)+\aleph(\Phi(x, p))], \\
B\left(\Phi(x, p), \frac{\partial \Phi(x, p)}{\partial x}\right)=0,
\end{gathered}
$$

where $x \in \mathbb{R}$ and $p \in[0,1]$ is an embedding parameter, $h(p)$ is a nonlinear auxiliary function for $p \neq 0, h(0)=0$ and $\Phi(x, p)$ is an unknown function. Clearly, when $p=0$ and $p=1$, it holds that $\Phi(x, 0)=v_{0}(x)$ and $\Phi(x, 1)=\widetilde{v}(x)$, respectively.

Thus, as $p$ varies from 0 to 1 , the solution $\Phi(x, p)$ approaches from $v_{0}(x)$ to $\widetilde{v}(x)$.

We choose the auxiliary function $h(p)$ in the form of

$$
h(p)=\sum_{k=0}^{m} p^{k} C_{k}
$$

where $C_{k}$ are convergence controlling constants to be determined.

To obtain an approximate solution, we expand $\Phi\left(x, p, C_{i}\right)$ in a Taylor series about $p$ as follows:

$$
\Phi\left(x, p, C_{i}\right)=v_{0}(x)+\sum_{k=1}^{m} v_{k}\left(C_{1}, C_{2}, \ldots, C_{k}\right) p^{k}
$$

Substituting (25) into (23) and equating the coefficients of like powers of $p$, we obtain the following equations.

The zeroth-order problem is

$$
\mathfrak{I}\left[v_{0}(x)\right]+f(x)=0, \quad B\left(v_{0}, \frac{d v_{0}}{d x}\right)=0
$$

First-order problem is

$$
\mathfrak{I}\left[v_{1}(x)\right]+f(x)=C_{1} \aleph_{0}\left[v_{0}(x)\right], \quad B\left(v_{1}, \frac{d v_{1}}{d x}\right)=0
$$

Second-order problem is

$$
\begin{gathered}
\mathfrak{I}\left[v_{2}(x)\right]+\mathfrak{I}\left[v_{1}(x)\right] \\
=C_{2} \aleph_{0}\left[v_{0}(x)\right]+C_{1}\left\{\mathfrak{J}\left[v_{1}(x)\right]+\aleph_{1}\left[v_{0}(x), v_{1}(x)\right]\right\}, \\
B\left(v_{2}, \frac{d v_{2}}{d x}\right)=0 .
\end{gathered}
$$


The general equations for $v_{k}(x)$ are given by

$$
\begin{gathered}
\mathfrak{I}\left[v_{k}(x)\right]-\mathfrak{J}\left[v_{k-1}(x)\right] \\
=C_{1} \aleph_{0}\left[v_{0}(x)\right] \\
+\sum_{i=1}^{k-1} C_{i}\left\{\mathfrak{I}\left[v_{k-i}(x)\right]\right. \\
\left.+\aleph_{k-i}\left[v_{0}(x), v_{1}(x), \ldots, v_{k-1}(x)\right]\right\}, \\
B\left(v_{k}, \frac{d v_{k}}{d x}\right)=0, \\
k=2,3, \ldots,
\end{gathered}
$$

where the coefficient of $p^{m}$ in the expansion of $\aleph(\Phi(x, p))$ about $p$ is $\aleph_{m}\left[v_{0}(x), v_{1}(x), \ldots, v_{m-1}(x)\right]$.

$$
\begin{aligned}
\aleph & \left(\Phi\left(x, p, C_{i}\right)\right) \\
& =\aleph_{0}\left[v_{0}(x)\right]+\sum_{m=1}^{m} \aleph_{m}\left[v_{0}(x), v_{1}(x), \ldots, v_{m-1}(x)\right] p^{m}
\end{aligned}
$$

It is noted that the convergence of the series (25) depends upon $C_{k}$. For convergence at $p=1$, the $m$ th order approximation $\widetilde{v}$ is

$$
\widetilde{v}\left(x, C_{1}, C_{2}, \ldots C_{m}\right)=v_{0}(x)+\sum_{j=1}^{m} v_{j}\left(x, C_{1}, C_{2}, \ldots C_{j}\right) .
$$

Substituting (31) in (22), the expression for residual is

$$
\begin{aligned}
& \mathfrak{R}\left(x, C_{1}, C_{2}, \ldots, C_{m}\right) \\
& =\mathfrak{J}\left[\widetilde{v}\left(x, C_{1}, C_{2}, \ldots, C_{m}\right)\right]+f(x) \\
& +\aleph\left[\widetilde{v}\left(x, C_{1}, C_{2}, \ldots, C_{m}\right)\right] .
\end{aligned}
$$

If $\Re=0$, then $\widetilde{v}$ will be the exact solution but usually this does not happen in nonlinear problems.

There are various methods to find the optimal values of $C_{i}, i=1,2, \ldots$. We apply the method of least square and Galerkin's method in the following manner:

In method of least square

$$
J\left(x, C_{1}, C_{2}, \ldots, C_{m}\right)=\int_{a}^{b} \Re^{2}\left(x, C_{1}, C_{2}, \ldots, C_{m}\right) d x .
$$

Minimizing $J\left(x, C_{1}, C_{2}, \ldots, C_{m}\right)$, we have

$$
\frac{\partial J}{\partial C_{i}}=0, \quad i=1,2, \ldots, m \text {. }
$$

In Galerkin's method, we solve the following system for $C_{i}(i=1,2, \ldots, m)$ :

$$
\int_{a}^{b} \mathfrak{R} \frac{\partial \widetilde{v}}{\partial C_{i}} d x=0, \quad i=1,2, \ldots, m
$$

To find appropriate $C_{i}(i=1,2, \ldots, m)$, we choose $a$ and $b$ in the domain of the problem. Approximate solution of order $m$ is well-determined with these known constants.

\section{Application of OHAM in Case of No-Slip Boundary}

Using (19) and (20) various order problems are as follows: Zeroth-order problem

$$
\begin{gathered}
v_{0}^{(i v)}(\eta)=0, \\
v_{0}(0)=0, \quad v_{0}^{\prime \prime}(0)=0, \quad v_{0}(1)=1, \quad v_{0}^{\prime}(1)=0 .
\end{gathered}
$$

First-order problem

$$
\begin{aligned}
& v_{1}^{(i v)}(\eta)= 3 C_{1} R v_{0}^{\prime \prime}(\eta)+C_{1} R \eta v_{0}^{\prime \prime \prime}(\eta)-C_{1} R v_{0}(\eta) v_{0}^{\prime \prime \prime}(\eta) \\
&+v_{0}^{(i v)}(\eta)+C_{1} v_{0}^{(i v)}(\eta), \\
& v_{1}(0)=0, \quad v_{1}^{\prime \prime}(0)=0, \quad v_{1}(1)=0, \quad v_{1}^{\prime}(1)=0 .
\end{aligned}
$$

Second-order problem

$$
\begin{array}{r}
v_{2}^{(i v)}(\eta)=3 C_{1} R v_{1}^{\prime \prime}(\eta)-C_{1} R v_{1}(\eta) v_{0}^{\prime \prime \prime}(\eta)+C_{1} R \eta v_{1}^{\prime \prime \prime}(\eta) \\
-C_{1} R v_{0}(\eta) v_{1}^{\prime \prime \prime}(\eta)+v_{1}^{(i v)}(\eta)+C_{1} v_{1}^{(i v)}(\eta), \\
v_{2}(0)=0, \quad v_{2}^{\prime \prime}(0)=0, \quad v_{2}(1)=0, \quad v_{2}^{\prime}(1)=0 .
\end{array}
$$

Third-order problem

$$
\begin{gathered}
v_{3}^{(i v)}(\eta)=3 C_{1} R v_{2}^{\prime \prime}(\eta)-C_{1} R v_{2}(\eta) v_{0}^{\prime \prime \prime}(\eta) \\
-C_{1} R v_{1}(\eta) v_{1}^{\prime \prime \prime}(\eta)+C_{1} R \eta v_{2}^{\prime \prime \prime}(\eta) \\
-C_{1} R v_{0}(\eta) v_{2}^{\prime \prime \prime}(\eta)+v_{2}^{(i v)}(\eta) \\
+C_{1} v_{2}^{(i v)}(\eta), \\
v_{3}(0)=0, \quad v_{3}^{\prime \prime}(0)=0, \quad v_{3}(1)=0, \quad v_{3}^{\prime}(1)=0 .
\end{gathered}
$$

Fourth-order problem

$$
\begin{aligned}
& v_{4}^{(i v)}(\eta)= 3 C_{1} R v_{3}^{\prime \prime}(\eta)-C_{1} R v_{3}(\eta) v_{0}^{\prime \prime \prime}(\eta) \\
&-C_{1} R v_{2}(\eta) v_{1}^{\prime \prime \prime}(\eta)-C_{1} R v_{1}(\eta) v_{2}^{\prime \prime \prime}(\eta) \\
&+C_{1} R \eta v_{3}^{\prime \prime \prime}(\eta)-C_{1} R v_{0}(\eta) v_{3}^{\prime \prime \prime}(\eta) \\
&+v_{3}^{(i v)}(\eta)+C_{1} v_{3}^{(i v)}(\eta), \\
& v_{4}(0)=0, \quad v_{4}^{\prime \prime}(0)=0, \quad v_{4}(1)=0, \quad v_{4}^{\prime}(1)=0 .
\end{aligned}
$$

By considering fourth-order solution, we have

$$
\widetilde{v}(\eta)=v_{0}(\eta)+v_{1}(\eta)+v_{2}(\eta)+v_{3}(\eta)+v_{4}(\eta) .
$$

The residual of the problem is

$$
\Re=\frac{d^{4} \widetilde{v}(\eta)}{d \eta^{4}}+R\left[(\eta-\widetilde{v}(\eta)) \frac{d^{3} \widetilde{v}(\eta)}{d \eta^{3}}+3 \frac{d^{2} \widetilde{v}(\eta)}{d \eta^{2}}\right] .
$$


We apply Galerkin's method to find constant $C_{1}$ as follows:

$$
\int_{0}^{1} \mathfrak{R} \frac{\partial \widetilde{v}}{\partial C_{1}} d x=0 .
$$

Solving (43) and keeping $R=0.1$, we get

$$
C_{1}=1.01328 \text {. }
$$

Using above value of $C_{1}$, the approximate solution is

$$
\begin{aligned}
\widetilde{v}(\eta)= & \frac{1}{2}\left(3 \eta-\eta^{3}\right) \\
+ & \frac{1}{560}\left(3.74914 \eta-7.39694 \eta^{3}+3.54648 \eta^{5}\right. \\
+ & \left.0.101328 \eta^{7}\right) \\
+ & \frac{1}{776160}\left(\begin{array}{l}
9.6941 \eta-430.222 \eta^{3}+514.14 \eta^{5} \\
+\frac{1}{565045}\left(2544.14 \eta+14364.4 \eta^{3}-37116.4 \eta^{5}\right.
\end{array}\right. \\
& +25023.2 \eta^{7}-8133.38 \eta^{9}+2599.01 \eta^{11} \\
& \left.+696.10 \eta^{13}+22.962 \eta^{15}\right) \\
+\frac{1}{722737}( & 1.53702 \eta-1.87989 \times 10^{8} \eta^{3} \\
& +1.93991 \times 10^{8} \eta^{5}+8.28837 \times 10^{7} \eta^{7} \\
& -8.03587 \times 10^{7} \eta^{9}-2.92844 \times 10^{7} \eta^{11} \\
& +1.67506 \times 10^{7} \eta^{13}-9.66217 \times 10^{6} \eta^{15} \\
& \left.-1.6529 \times 10^{6} \eta^{17}-48038.9 \eta^{19}\right)
\end{aligned}
$$

\section{Application of OHAM in Case of Slip Boundary}

Using (19) and (21) different order problems are as follows. Zeroth-order problem

$$
\begin{gathered}
u_{0}^{(i v)}(\eta)=0 \\
u_{0}(0)=0, \quad u_{0}^{\prime \prime}(0)=0, \quad u_{0}(1)=1, \quad u_{0}^{\prime}(1)=\gamma u_{0}^{\prime \prime}(1) .
\end{gathered}
$$

First-order problem

$$
\begin{aligned}
u_{1}^{(i v)}(\eta) & \\
= & 3 C_{1} R u_{0}^{\prime \prime}(\eta)+C_{1} R \eta u_{0}^{\prime \prime \prime}(\eta)-C_{1} R u_{0}(\eta) u_{0}^{\prime \prime \prime}(\eta) \\
& +u_{0}^{(i v)}(\eta)+C_{1} u_{0}^{(i v)}(\eta), \\
u_{1}(0)= & 0, \quad u_{1}^{\prime \prime}(0)=0, \quad u_{1}(1)=0, \quad u_{1}^{\prime}(1)=\gamma u_{1}^{\prime \prime}(1) .
\end{aligned}
$$

Second-order problem

$$
\begin{aligned}
& u_{2}^{(i v)}(\eta)= 3 C_{1} R u_{1}^{\prime \prime}(\eta)-C_{1} R u_{1}(\eta) u_{0}^{\prime \prime \prime}(\eta) \\
&+C_{1} R \eta u_{1}^{\prime \prime \prime}(\eta)-C_{1} R u_{0}(\eta) u_{1}^{\prime \prime \prime}(\eta) \\
&+u_{1}^{(i v)}(\eta)+C_{1} u_{1}^{(i v)}(\eta) \\
& u_{2}(0)=0, \quad u_{2}^{\prime \prime}(0)=0, \quad u_{2}(1)=0, \quad u_{2}^{\prime}(1)=\gamma u_{2}^{\prime \prime}(1) .
\end{aligned}
$$

Third-order problem

$$
\begin{aligned}
& u_{3}^{(i v)}(\eta)= 3 C_{1} R u_{2}^{\prime \prime}(\eta)-C_{1} R u_{2}(\eta) u_{0}^{\prime \prime \prime}(\eta) \\
&-C_{1} R u_{1}(\eta) u_{1}^{\prime \prime \prime}(\eta)+C_{1} R \eta u_{2}^{\prime \prime \prime}(\eta) \\
&-C_{1} R u_{0}(\eta) u_{2}^{\prime \prime \prime}(\eta)+u_{2}^{(i v)}(\eta) \\
&+C_{1} u_{2}^{(i v)}(\eta), \\
& u_{3}(0)=0, \quad u_{3}^{\prime \prime}(0)=0, \quad u_{3}(1)=0, \quad u_{3}^{\prime}(1)=\gamma u_{3}^{\prime \prime}(1) .
\end{aligned}
$$

Fourth-order problem

$$
\begin{aligned}
& u_{4}^{(i v)}(\eta)= 3 C_{1} R u_{3}^{\prime \prime}(\eta)-C_{1} R u_{3}(\eta) u_{0}^{\prime \prime \prime}(\eta) \\
&-C_{1} R u_{2}(\eta) u_{1}^{\prime \prime \prime}(\eta)-C_{1} R u_{1}(\eta) u_{2}^{\prime \prime \prime}(\eta) \\
&+C_{1} R \eta u_{3}^{\prime \prime \prime}(\eta)-C_{1} R u_{0}(\eta) u_{3}^{\prime \prime \prime}(\eta) \\
&+u_{3}^{(i v)}(\eta)+C_{1} u_{3}^{(i v)}(\eta), \\
& u_{4}(0)=0, \quad u_{4}^{\prime \prime}(0)=0, \quad u_{4}(1)=0, \quad u_{4}^{\prime}(1)=\gamma u_{4}^{\prime \prime}(1) .
\end{aligned}
$$

By considering fourth-order solution, we have

$$
\widetilde{u}(\eta)=\sum_{i=0}^{4} u_{i}\left(\eta, C_{1}\right)
$$

The residual of the problem is

$$
\zeta=\frac{d^{4} \tilde{u}(\eta)}{d \eta^{4}}+R\left[(\eta-\tilde{u}(\eta)) \frac{d^{3} \widetilde{u}(\eta)}{d \eta^{3}}+3 \frac{d^{2} \widetilde{u}(\eta)}{d \eta^{2}}\right] .
$$

We apply Galerkin's method to find constant $C_{1}$ as follows:

$$
\int_{0}^{1} \zeta \frac{\partial \widetilde{u}}{\partial C_{1}} d x=0
$$

Solving (53) and taking $R=0.2$ and $\gamma=1$, we get

$$
C_{1}=-1.06872
$$


TABLE 1: OHAM solutions along with residuals for various $R$ in case of no-slip boundary.

\begin{tabular}{|c|c|c|c|c|c|c|}
\hline \multirow{2}{*}{$\eta$} & \multicolumn{2}{|c|}{$R=0.1$} & \multicolumn{2}{|c|}{$R=0.3$} & \multicolumn{2}{|c|}{$R=0.5$} \\
\hline & Solution & Residual & Solution & Residual & Solution & Residual \\
\hline 0.0 & 0. & 0. & 0. & 0. & 0. & 0. \\
\hline 0.1 & 0.150158 & $5.37334 \times 10^{-11}$ & 0.151534 & $-6.69283 \times 10^{-9}$ & 0.152999 & $-6.23412 \times 10^{-8}$ \\
\hline 0.2 & 0.297237 & $3.14675 \times 10^{-10}$ & 0.299827 & $1.66025 \times 10^{-9}$ & 0.302582 & $6.58388 \times 10^{-8}$ \\
\hline 0.3 & 0.438170 & $8.51847 \times 10^{-10}$ & 0.441661 & $2.33504 \times 10^{-8}$ & 0.445373 & $3.42092 \times 10^{-7}$ \\
\hline 0.4 & 0.569900 & $1.51431 \times 10^{-9}$ & 0.573869 & $3.93945 \times 10^{-8}$ & 0.578082 & $4.93942 \times 10^{-7}$ \\
\hline 0.5 & 0.689397 & $1.90824 \times 10^{-9}$ & 0.693354 & $2.81614 \times 10^{-8}$ & 0.697548 & $2.42092 \times 10^{-7}$ \\
\hline 0.6 & 0.793661 & $1.33301 \times 10^{-9}$ & 0.797122 & $-3.19654 \times 10^{-8}$ & 0.800780 & $-6.53046 \times 10^{-7}$ \\
\hline 0.7 & 0.879734 & $-1.59255 \times 10^{-9}$ & 0.882300 & $-2.21911 \times 10^{-7}$ & 0.885004 & $-3.23415 \times 10^{-6}$ \\
\hline 0.8 & 0.944705 & $-1.02814 \times 10^{-8}$ & 0.946167 & $-8.84707 \times 10^{-7}$ & 0.947702 & $-1.2206 \times 10-5$ \\
\hline 0.9 & 0.985722 & $-3.446 \times 10^{-8}$ & 0.986180 & $-3.21268 \times 10^{-6}$ & 0.986659 & $-4.41008 \times 10^{-5}$ \\
\hline 1.0 & 1. & $-1.03509 \times 10^{-7}$ & 1. & $-1.11826 \times 10^{-5}$ & 1. & $-1.54022 \times 10^{-4}$ \\
\hline
\end{tabular}

Using above value of $C_{1}$, the approximate solution is

$$
\begin{aligned}
& \tilde{u}(\eta)=\frac{1}{2}\left(3 \eta+\eta^{3}\right) \\
& +\frac{1}{4480}\left(-113.284 \eta+151.758 \eta^{3}-38.9012 \eta^{5}\right. \\
& \left.+0.427486 \eta^{7}\right) \\
& +\frac{1}{248372}\left(-156189 . \eta+273825 . \eta^{3}-160808 . \eta^{5}\right. \\
& \left.+45952.6 \eta^{7}-2814.27 \eta^{9}+34.5387 \eta^{11}\right) \\
& +\frac{1}{723257}\left(-9.83844 \eta+1.149 \times 10^{8} \eta^{3}\right. \\
& -3.35388 \times 10^{7} \eta^{5}+4.04185 \times 10^{7} \eta^{7} \\
& -2.68964 \times 10^{7} \eta^{9}+3.65673 \times 10^{6} \eta^{11} \\
& \left.-157538 . \eta^{13}+1724.2 \eta^{15}\right) \\
& +\frac{1}{370042}\left(-1.97195 \times 10^{13} \eta+4.25372 \times 10^{13} \eta^{3}\right. \\
& -2.94213 \times 10^{13} \eta^{5}+6.06765 \times 10^{12} \eta^{7} \\
& -1.39445 \times 10^{12} \eta^{9}+2.47846 \times 10^{12} \eta^{11} \\
& -5.97188 \times 10^{11} \eta^{13}+5.08263 \times 10^{10} \eta^{15} \\
& \left.-1.62422 \times 10^{9} \eta^{17}+1.52182 \times 10^{7} \eta^{19}\right) \text {. }
\end{aligned}
$$

\section{Results and Discussions}

In this article we considered the unsteady axisymmetric flow of nonconducting, incompressible Newtonian fluid between two circular plates. The resulting nonlinear boundary value problems are solved with OHAM and fourth-order RungeKutta method using Mathematica 7.0.

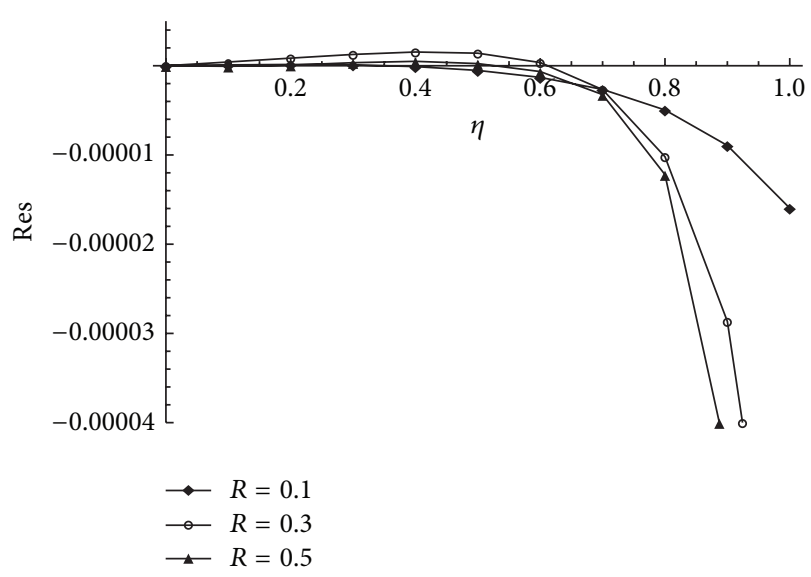

FigURE 2: OHAM residuals at various values of $R$ in case of no-slip boundary.

Tables 1, 3, and 5 reflect OHAM solutions along with residuals in case of no-sip and slip boundaries for various values of Reynolds number $R$ and slip parameter $\gamma$. Also, Tables 2, 4, and 6 represent RK4 solutions along with residuals in case of no-slip and slip boundaries for various values of $R$ and $\gamma$. All the tables demonstrate that results obtained using OHAM are in agreement with RK4 by means of residuals. In addition to above mentioned tables, Table 7 shows the comparison of solutions obtained from OHAM and RK4 for various values of Reynolds number $R$.

Furthermore, Figures 2, 3, and 4 indicate the OHAM residuals in case of no-slip and slip boundaries for various values of $R$ and $\gamma$.

The effect of Reynolds number $R$ on velocity profiles in case of no-slip boundary is shown in Figure 5. In these profiles we varied $R$ as $R=0.1,1,2,3$ and observed that the normal velocity is increased with the increase of Reynolds number (Figure 5(a)). It is also noted that the normal velocity monotonically increases from $\eta=0$ to $\eta=1$ for fixed positive value of $R$ at a given time. Figure 5(b) describes the impact of $R$ on the longitudinal velocity in case of no-slip boundary. It 
TABLE 2: RK4 solutions along with residuals for various $R$ in case of no-slip boundary.

\begin{tabular}{lccccrr}
\hline$\eta$ & \multicolumn{2}{c}{$R=0.1$} & \multicolumn{2}{c}{$R=0.3$} & \multicolumn{2}{c}{$R=0.5$} \\
& Solution & Residual & Solution & Residual & Solution & 0. \\
0.0 & 0. & $2.88535 \times 10^{-7}$ & 0. & $9.66906 \times 10^{-5}$ & $5.30549 \times 10^{-4}$ \\
0.1 & 0.150158 & $3.88278 \times 10^{-8}$ & 0.151534 & $5.71393 \times 10^{-6}$ & 0.152999 & $3.04600 \times 10^{-5}$ \\
0.2 & 0.297237 & $-9.97789 \times 10^{-9}$ & 0.299827 & $-1.41434 \times 10^{-6}$ & 0.302582 & $-7.52772 \times 10^{-6}$ \\
0.3 & 0.438170 & $2.93767 \times 10^{-9}$ & 0.441661 & $3.95997 \times 10^{-7}$ & 0.445373 & $2.10296 \times 10^{-6}$ \\
0.4 & 0.569900 & $-7.51436 \times 10^{-10}$ & 0.573869 & $-1.15203 \times 10^{-7}$ & 0.578082 & $-6.18081 \times 10^{-7}$ \\
0.5 & 0.689397 & $-4.71705 \times 10^{-10}$ & 0.693354 & $-9.6761 \times 10^{-9}$ & 0.697548 & $-2.95916 \times 10^{-8}$ \\
0.6 & 0.793661 & $2.23004 \times 10^{-9}$ & 0.797122 & $1.4593 \times 10^{-7}$ & 0.800780 & $7.14133 \times 10^{-7}$ \\
0.7 & 0.879734 & $-7.18245 \times 10^{-9}$ & 0.882300 & $-4.86272 \times 10^{-7}$ & 0.885004 & $-2.39245 \times 10^{-6}$ \\
0.8 & 0.944705 & $2.68744 \times 10^{-8}$ & 0.946167 & $1.77796 \times 10^{-6}$ & 0.947702 & $8.70664 \times 10^{-6}$ \\
0.9 & 0.985722 & $-1.11599 \times 10^{-7}$ & 0.986180 & $-7.28952 \times 10^{-6}$ & 0.986659 & $3.56157 \times 10^{-5}$ \\
1.0 & 1. & $-2.71683 \times 10^{-6}$ & 1. & $-1.47695 \times 10^{-4}$ & 1. & $6.90088 \times 10^{-4}$ \\
\hline
\end{tabular}

TABLE 3: OHAM solutions along with residuals for various values of $R$ in case of slip boundary.

\begin{tabular}{|c|c|c|c|c|c|c|}
\hline \multirow{2}{*}{$\eta$} & \multicolumn{2}{|c|}{$R=0.2$} & \multicolumn{2}{|c|}{$R=0.3$} & \multicolumn{2}{|c|}{$R=0.4$} \\
\hline & Solution & Residual & Solution & Residual & Solution & Residual \\
\hline 0.0 & 0. & 0. & 0. & 0. & 0. & 0. \\
\hline 0.1 & 0.072692 & $3.18345 \times 10^{-8}$ & 0.071220 & $3.15621 \times 10^{-7}$ & 0.069593 & $1.74626 \times 10^{-6}$ \\
\hline 0.2 & 0.147091 & $-1.72789 \times 10^{-8}$ & 0.144268 & $-9.14579 \times 10^{-8}$ & 0.141147 & $-1.13148 \times 10^{-7}$ \\
\hline 0.3 & 0.224893 & $-1.96995 \times 10^{-7}$ & 0.220953 & $-1.66461 \times 10^{-6}$ & 0.216599 & $-7.79061 \times 10^{-6}$ \\
\hline 0.4 & 0.307773 & $-4.94482 \times 10^{-7}$ & 0.303048 & $-4.29173 \times 10^{-6}$ & 0.297832 & $-2.07401 \times 10^{-5}$ \\
\hline 0.5 & 0.397370 & $-7.99437 \times 10^{-7}$ & 0.392277 & $-6.99917 \times 10^{-6}$ & 0.386657 & $-3.41643 \times 10^{-5}$ \\
\hline 0.6 & 0.495283 & $-8.5784 \times 10^{-7}$ & 0.490289 & $-7.5499 \times 10^{-6}$ & 0.484784 & $-3.70689 \times 10^{-5}$ \\
\hline 0.7 & 0.603056 & $-2.1053 \times 10^{-7}$ & 0.598654 & $-1.91141 \times 10^{-6}$ & 0.593805 & $-9.6958 \times 10^{-6}$ \\
\hline 0.8 & 0.722172 & $1.87065 \times 10^{-6}$ & 0.718841 & $1.62936 \times 10^{-5}$ & 0.715176 & $7.91082 \times 10^{-5}$ \\
\hline 0.9 & 0.854042 & $6.40576 \times 10^{-6}$ & 0.852211 & $5.59717 \times 10^{-5}$ & 0.850196 & $2.72708 \times 10^{-4}$ \\
\hline 1.0 & 1. & $1.46076 \times 10^{-5}$ & 1. & $1.27671 \times 10^{-4}$ & 1. & $6.22242 \times 10^{-4}$ \\
\hline
\end{tabular}

TABLE 4: RK4 solutions along with residuals for various values of $R$ in case of slip boundary.

\begin{tabular}{lcccccc}
\hline$\eta$ & \multicolumn{3}{c}{$R=0.2$} & \multicolumn{2}{c}{$R=0.3$} & \multicolumn{2}{c}{$R=0.4$} \\
& Solution & Residual & Solution & Residual & Solution & \multicolumn{2}{c}{ Residual } \\
\hline 0.0 & 0. & $4.12179 \times 10^{-6}$ & 0. & $7.56765 \times 10^{-6}$ & $0.62897 \times 10^{-6}$ \\
0.1 & 0.072692 & $1.74999 \times 10^{-7}$ & 0.071220 & $2.16125 \times 10^{-7}$ & 0.069593 & $-3.49994 \times 10^{-7}$ \\
0.2 & 0.147091 & $-4.21503 \times 10^{-8}$ & 0.144268 & $-4.97423 \times 10^{-8}$ & 0.141147 & $9.53266 \times 10^{-8}$ \\
0.3 & 0.224893 & $1.12883 \times 10^{-8}$ & 0.220953 & $1.22294 \times 10^{-8}$ & 0.216598 & $-3.0735 \times 10^{-8}$ \\
0.4 & 0.307773 & $-3.52272 \times 10^{-9}$ & 0.303048 & $-4.30187 \times 10^{-9}$ & 0.297832 & $7.2493 \times 10^{-9}$ \\
0.5 & 0.397370 & $7.80684 \times 10^{-10}$ & 0.392276 & $3.06668 \times 10^{-9}$ & 0.386657 & $8.46409 \times 10^{-9}$ \\
0.6 & 0.495283 & $1.10391 \times 10^{-9}$ & 0.490289 & $-5.28586 \times 10^{-9}$ & 0.484783 & $-3.36828 \times 10^{-8}$ \\
0.7 & 0.603056 & $-4.35033 \times 10^{-9}$ & 0.598654 & $1.54159 \times 10^{-8}$ & 0.593805 & $1.06816 \times 10^{-7}$ \\
0.8 & 0.722172 & $1.44198 \times 10^{-8}$ & 0.718841 & $-6.05873 \times 10^{-8}$ & 0.715175 & $-3.98519 \times 10^{-7}$ \\
0.9 & 0.854042 & $-5.53933 \times 10^{-8}$ & 0.852211 & $2.59233 \times 10^{-7}$ & 0.850196 & $1.65518 \times 10^{-6}$ \\
1.0 & 1. & $-1.97434 \times 10^{-7}$ & 1. & $7.96168 \times 10^{-6}$ & 1. & $3.89126 \times 10^{-5}$ \\
\hline
\end{tabular}

is experienced that this component of velocity deceases near the wall but increases near the central axis of the channel.

The effect of Reynolds number $R$ on velocity profiles in case of slip boundary is depicted in Figure 6. In these profiles, we fixed slip parameter $\gamma=1$ and varied Reynolds number $R$ as $R=0.2,0.6,1,1.5$. It is noted that the normal velocity decreases as the Reynolds number increases (Figure 6(a)). It is also observed that longitudinal velocity decreases near the central axis of the channel but increases near the walls when $R$ increases (Figure 6(b)).

Figure 7 demonstrates the effect of slip parameter $\gamma$ on the velocity profiles. After fixing Reynolds number $R=0.3$ we varied $\gamma$ as $\gamma=0.6,0.7,0.8,1$. We find that normal velocity increases as $\gamma$ increases. It is also noted that longitudinal velocity decreases near the walls but increases near central axis of the channel. 
TABLE 5: OHAM solutions along with residuals for various values of $\gamma$ in case of slip boundary.

\begin{tabular}{|c|c|c|c|c|c|c|}
\hline \multirow{2}{*}{$\eta$} & \multicolumn{2}{|c|}{$\gamma=0.5$} & \multicolumn{2}{|c|}{$\gamma=0.6$} & \multicolumn{2}{|c|}{$\gamma=0.7$} \\
\hline & Solution & Residual & Solution & Residual & Solution & Residual \\
\hline 0.0 & 0. & 0. & 0. & 0. & 0. & 0. \\
\hline 0.1 & -0.00880011 & $-1.07933 \times 10^{-6}$ & 0.0336596 & $-7.54858 \times 10^{-8}$ & 0.0522402 & $-7.74445 \times 10^{-9}$ \\
\hline 0.2 & -0.010881 & $-2.2445 \times 10^{-6}$ & 0.0714091 & $-1.73419 \times 10^{-7}$ & 0.1074223 & $-2.51485 \times 10^{-8}$ \\
\hline 0.3 & 0.000449012 & $-3.45224 \times 10^{-6}$ & 0.117324 & $-3.00407 \times 10^{-7}$ & 0.168479 & $-5.68899 \times 10^{-8}$ \\
\hline 0.4 & 0.0318278 & $-4.41058 \times 10^{-6}$ & 0.175449 & $-4.32397 \times 10^{-7}$ & 0.238322 & $-9.8056 \times 10^{-8}$ \\
\hline 0.5 & 0.0898133 & $-4.47787 \times 10^{-6}$ & 0.249785 & $-5.00627 \times 10^{-7}$ & 0.319832 & $-1.29675 \times 10^{-7}$ \\
\hline 0.6 & 0.18086 & $-2.60114 \times 10^{-6}$ & 0.344278 & $-3.77978 \times 10^{-7}$ & 0.415852 & $-1.13733 \times 10^{-7}$ \\
\hline 0.7 & 0.311298 & $2.65136 \times 10^{-6}$ & 0.462801 & $1.30706 \times 10^{-7}$ & 0.529175 & $1.20785 \times 10^{-8}$ \\
\hline 0.8 & 0.487312 & $1.28583 \times 10^{-5}$ & 0.609145 & $1.28166 \times 10^{-6}$ & 0.662538 & $3.36724 \times 10^{-7}$ \\
\hline 0.9 & 0.714929 & $2.91822 \times 10^{-5}$ & 0.787012 & $3.34515 \times 10^{-6}$ & 0.818613 & $9.67763 \times 10^{-7}$ \\
\hline 1.0 & 1. & $5.14019 \times 10^{-5}$ & 1. & $6.49074 \times 10^{-6}$ & 1. & $2.00176 \times 10^{-6}$ \\
\hline
\end{tabular}

TABLE 6: RK4 solutions along with residuals for various values of $\gamma$ in case of slip boundary.

\begin{tabular}{lcccccc}
\hline$\eta$ & Solution & Residual & Solution & Residual & Solution & \multicolumn{2}{c}{$\gamma=0.7$} \\
\hline 0.0 & 0. & $2.04594 \times 10^{-5}$ & 0. & $7.34794 \times 10^{-6}$ & 0.6 & $3.56291 \times 10^{-6}$ \\
0.1 & -0.00880012 & $7.72953 \times 10^{-7}$ & 0.0336596 & $3.21066 \times 10^{-7}$ & 0.0522402 & $1.63598 \times 10^{-7}$ \\
0.2 & -0.0108811 & $-1.8406 \times 10^{-7}$ & 0.0714091 & $-7.75305 \times 10^{-8}$ & 0.1074223 & $-3.96814 \times 10^{-8}$ \\
0.3 & 0.000448983 & $4.82747 \times 10^{-8}$ & 0.117324 & $2.08509 \times 10^{-8}$ & 0.168479 & $1.0753 \times 10^{-8}$ \\
0.4 & 0.0318277 & $-1.54235 \times 10^{-8}$ & 0.175449 & $-6.43999 \times 10^{-9}$ & 0.238322 & $-3.28063 \times 10^{-9}$ \\
0.5 & 0.0898132 & $5.14031 \times 10^{-9}$ & 0.249785 & $1.21029 \times 10^{-9}$ & 0.319832 & $4.50154 \times 10^{-10}$ \\
0.6 & 0.18086 & $-6.51866 \times 10^{-10}$ & 0.344278 & $2.64494 \times 10^{-9}$ & 0.415852 & $1.84079 \times 10^{-9}$ \\
0.7 & 0.311298 & $-1.86837 \times 10^{-9}$ & 0.462801 & $-9.88382 \times 10^{-9}$ & 0.529175 & $-6.51979 \times 10^{-9}$ \\
0.8 & 0.487312 & $-1.33003 \times 10^{-9}$ & 0.609145 & $3.37336 \times 10^{-8}$ & 0.662538 & $2.27322 \times 10^{-8}$ \\
0.9 & 0.714929 & $2.66400 \times 10^{-8}$ & 0.787012 & $-1.32232 \times 10^{-7}$ & 0.818613 & $-9.05714 \times 10^{-8}$ \\
1.0 & 1. & $5.55277 \times 10^{-6}$ & 1. & $-1.1671 \times 10^{-6}$ & & 1. \\
\hline
\end{tabular}

TABLE 7: Comparison of OHAM and RK4 solutions for various $R$ in case of slip and no-slip boundary.

\begin{tabular}{|c|c|c|c|c|c|c|}
\hline \multirow[t]{2}{*}{$\eta$} & \multicolumn{3}{|c|}{$\begin{array}{c}\text { In case of no-slip boundary } \\
\text { |RK4 Solution - OHAM Solution| }\end{array}$} & \multicolumn{3}{|c|}{$\begin{array}{c}\text { In case of slip boundary } \\
\mid \text { RK4 Solution - OHAM Solution| }\end{array}$} \\
\hline & $R=0.1$ & $R=0.3$ & $R=0.5$ & $R=0.2$ & $R=0.3$ & $R=0.4$ \\
\hline 0.0 & 0. & 0. & 0. & 0. & 0. & 0. \\
\hline 0.1 & $2.33147 \times 10^{-15}$ & $1.62249 \times 10^{-10}$ & $2.41725 \times 10^{-9}$ & $8.44379 \times 10^{-10}$ & $7.90896 \times 10^{-9}$ & $4.1479 \times 10^{-8}$ \\
\hline 0.2 & $9.49241 \times 10^{-14}$ & $3.14228 \times 10^{-10}$ & $4.67809 \times 10^{-9}$ & $1.6672 \times 10^{-9}$ & $1.55832 \times 10^{-8}$ & $8.15527 \times 10^{-8}$ \\
\hline 0.3 & $3.82361 \times 10^{-13}$ & $4.46143 \times 10^{-10}$ & $6.63065 \times 10^{-9}$ & $2.44889 \times 10^{-9}$ & $2.28102 \times 10^{-8}$ & $1.18952 \times 10^{-7}$ \\
\hline 0.4 & $9.13492 \times 10^{-13}$ & $5.47932 \times 10^{-10}$ & $8.11705 \times 10^{-9}$ & $3.1663 \times 10^{-9}$ & $2.93539 \times 10^{-8}$ & $1.52339 \times 10^{-7}$ \\
\hline 0.5 & $1.65451 \times 10^{-12}$ & $6.07476 \times 10^{-10}$ & $8.95202 \times 10^{-9}$ & $3.77514 \times 10^{-9}$ & $3.48026 \times 10^{-8}$ & $1.79574 \times 10^{-7}$ \\
\hline 0.6 & $2.42317 \times 10^{-12}$ & $6.09434 \times 10^{-10}$ & $8.91445 \times 10^{-9}$ & $4.18241 \times 10^{-9}$ & $3.8327 \times 10^{-8}$ & $1.96532 \times 10^{-7}$ \\
\hline 0.7 & $2.86218 \times 10^{-12}$ & $5.36833 \times 10^{-10}$ & $7.77928 \times 10^{-9}$ & $4.22088 \times 10^{-9}$ & $3.84569 \times 10^{-8}$ & $1.96009 \times 10^{-7}$ \\
\hline 0.8 & $2.52039 \times 10^{-12}$ & $3.78563 \times 10^{-10}$ & $5.4278 \times 10^{-9}$ & $3.65208 \times 10^{-9}$ & $3.31085 \times 10^{-8}$ & $1.67861 \times 10^{-7}$ \\
\hline 0.9 & $1.20232 \times 10^{-12}$ & $1.54202 \times 10^{-10}$ & $2.18716 \times 10^{-9}$ & $2.24454 \times 10^{-9}$ & $2.02732 \times 10^{-8}$ & $1.02385 \times 10^{-7}$ \\
\hline 1.0 & $1.26281 \times 10^{-16}$ & $2.53545 \times 10^{-16}$ & $2.93337 \times 10^{-17}$ & $1.7436 \times 10^{-14}$ & $4.92715 \times 10^{-14}$ & $3.8179 \times 10^{-14}$ \\
\hline
\end{tabular}

\section{Conclusions}

In this article, we find the similarity solution for unsteady axisymmetric squeezing flow of incompressible Newtonian fluid between two circular plates. We observed that the similarity solution exists only when distance between the plates varies as $(W t+S)^{1 / 2}$, and squeezing flow occurs when
$W<0, S>0$ and $(W t+S)>0$. The key findings of the present analysis are as follows:

In case of no-slip at boundary;

(i) It has been found that increase in Reynolds number $R$ increases the normal velocity. 


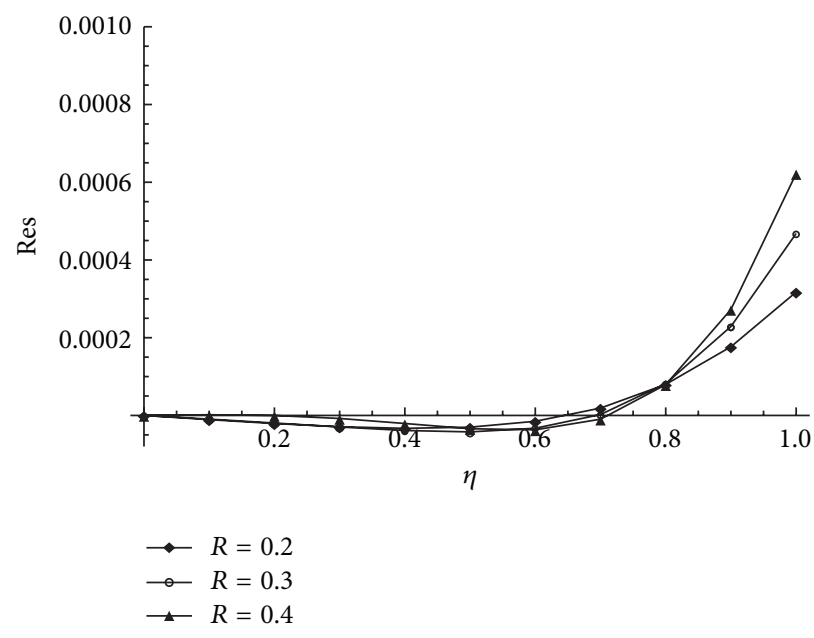

FiguRE 3: OHAM residuals at various values of $R$ in case of slip boundary.

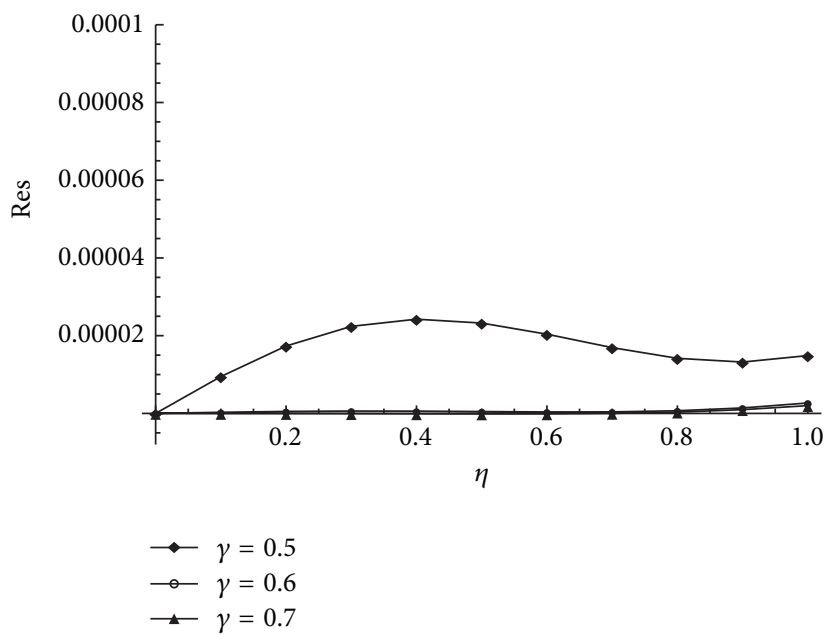

FIGURE 4: OHAM residuals at various values of $\gamma$ in case of slip boundary.

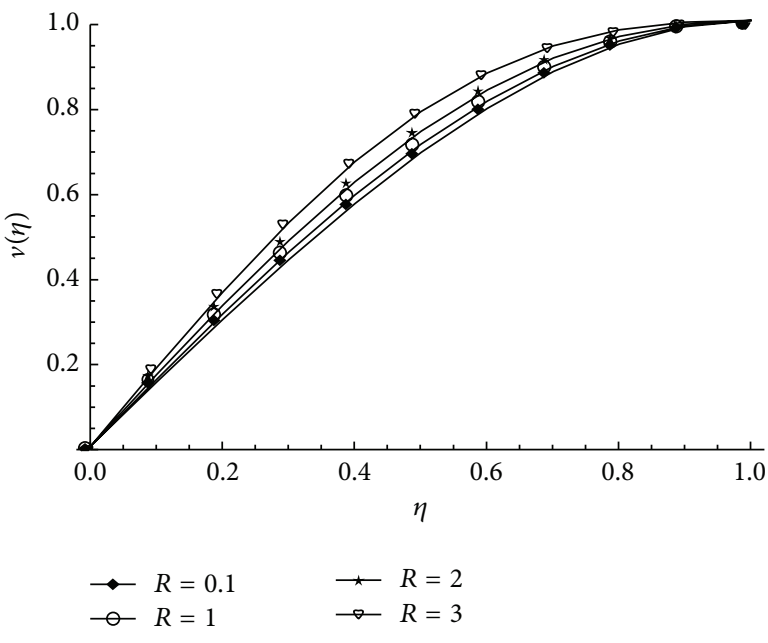

(a) The effect of $R$ on the Normal velocity profiles

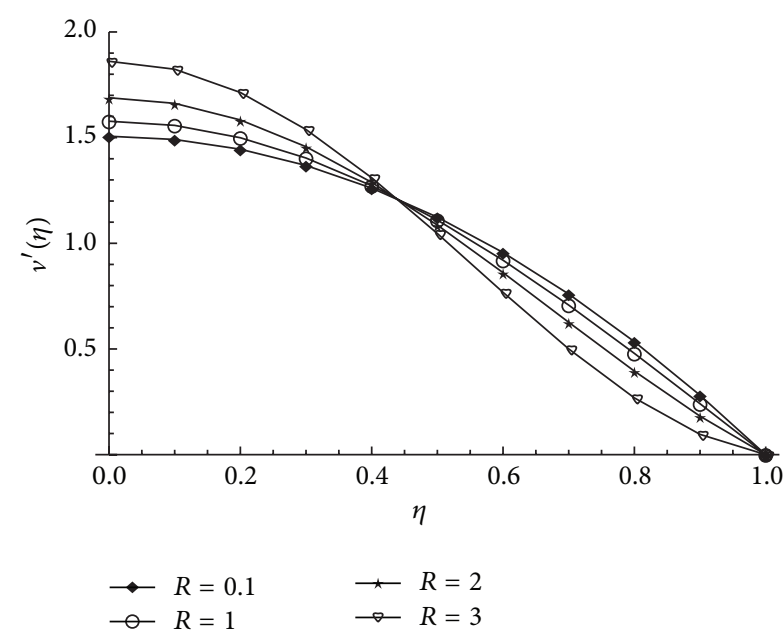

(b) The effect of $R$ on the longitudinal velocity profiles

FIGURE 5: Velocity profiles for various values of $R=0.1,1,2,3$ in case of no-slip boundary. 


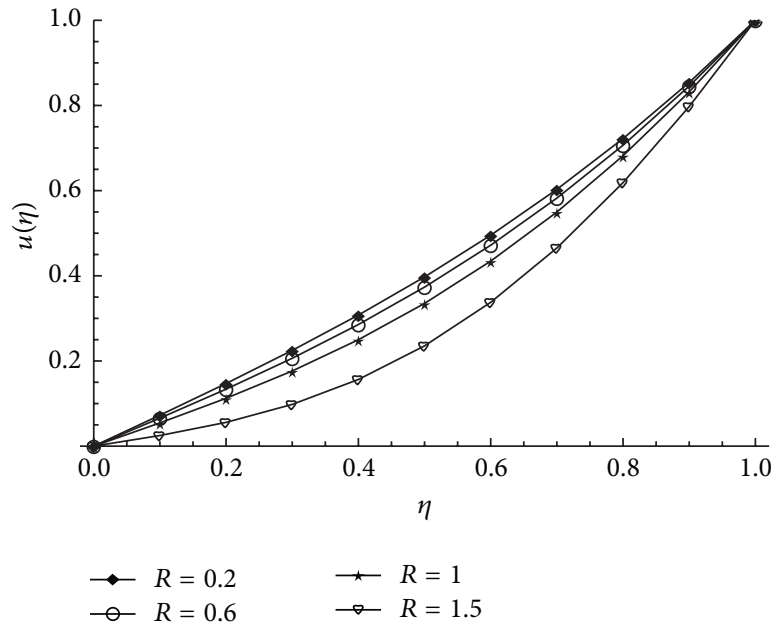

(a) The effect of $R$ on the Normal velocity profiles

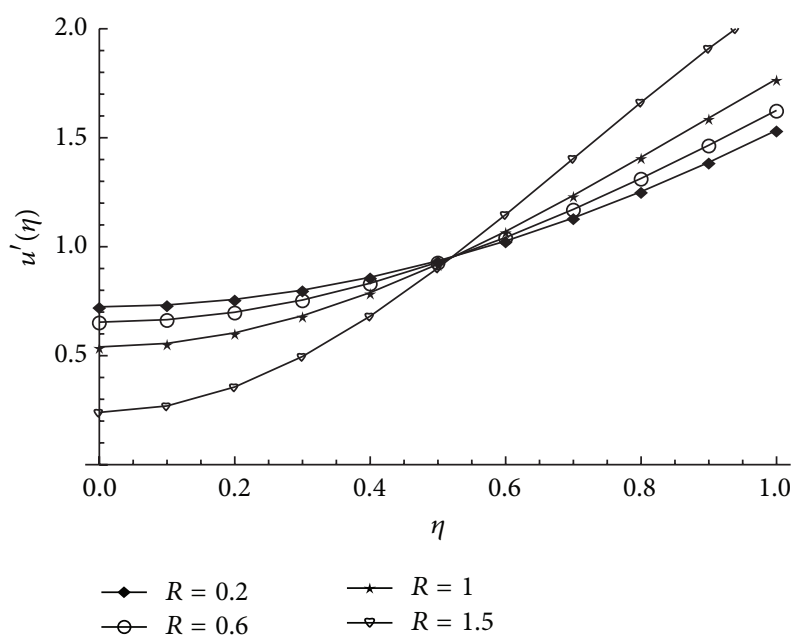

(b) The effect of $R$ on the longitudinal velocity profiles

FIGURE 6: Velocity profiles for various values of $R=0.2,0.6,1,1.5$ fixing $\gamma=1$ in case of slip boundary.

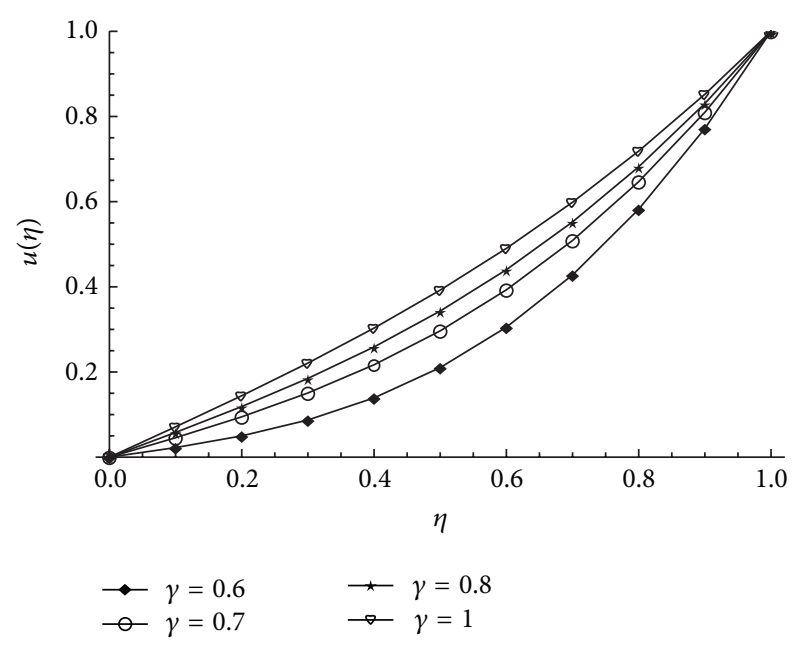

(a) The effect of $\gamma$ on the Normal velocity profiles

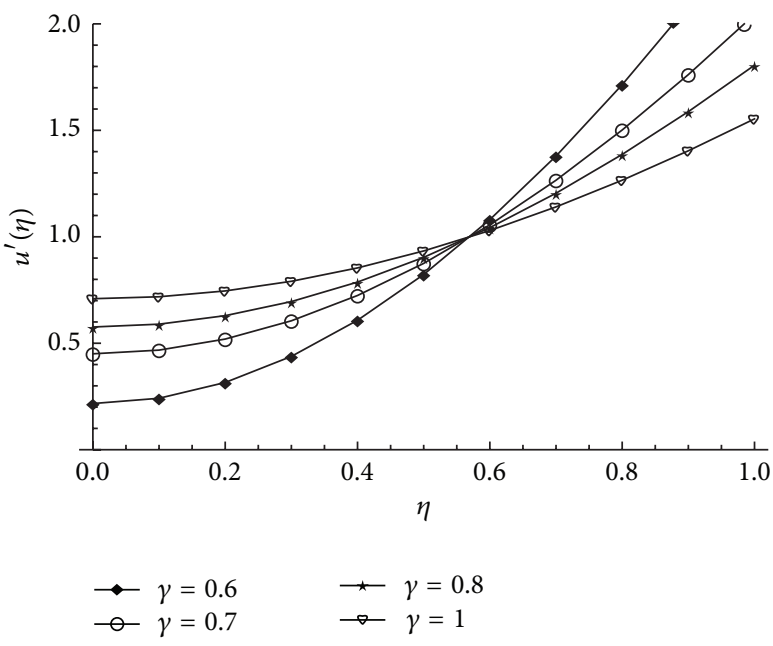

(b) The effect of $\gamma$ on the longitudinal velocity profiles

Figure 7: Velocity profiles for various values of $\gamma=0.6,0.7,0.8,1$ fixing $R=0.3$ in case of slip boundary.

(ii) It has been observed that normal velocity increases monotonically from $\eta=0$ to $\eta=1$ for fixed positive value of $R$ at a given time.

(iii) It has been seen that longitudinal velocity deceases near the walls and increases near the central axis of the channel.

In case of slip at boundary;

(i) It has been noted that after fixing slip parameter $\gamma$ and varying the Reynolds number $R$, the normal velocity profile decreases with the increase in $R$. Also the longitudinal velocity increases near the walls but decreases near the central axis of the channel.

(ii) It has been examined that for a fixed Reynolds number $R$ when we vary slip parameter $\gamma$, the normal velocity increases with the increase in $\gamma$. Also the longitudinal velocity decreases near the walls and increases near the central axis of the channel.

(iii) It has been investigated that Reynolds number $R$ and slip parameter $\gamma$ have opposite effects on the normal and longitudinal velocity components.

In case of slip versus no-slip boundary;

(i) It has been observed that Reynolds number $R$ has opposite behavior on the normal velocity in case of slip and no-slip boundaries.

(ii) It has been also noticed that Reynolds number $R$ has opposite effect on the longitudinal velocity near the central axis of the channel, while near the wall longitudinal velocity increases in case of slip boundary and decease in no-slip boundary. This is in conformance to [33]. 


\section{Conflict of Interests}

The authors declare that there is no conflict of interests regarding the publication of this paper.

\section{References}

[1] Q. K. Ghori, M. Ahmed, and A. M. Siddiqui, "Application of homotopy perturbation method to squeezing flow of a newtonian fluid," International Journal of Nonlinear Sciences and Numerical Simulation, vol. 8, no. 2, pp. 179-184, 2007.

[2] X. J. Ran, Q. Y. Zhu, and Y. Li, "An explicit series solution of the squeezing flow between two infinite plates by means of the homotopy analysis method," Communications in Nonlinear Science and Numerical Simulation, vol. 14, no. 1, pp. 119-132, 2009.

[3] M. J. Stefan, "Versuch Über die scheinbare adhäsion," Sitzungsberichte/Österreichische Akademie der Wissenschaften in Wien Mathematisch-Naturwissenschaftliche Klasse, vol. 69, pp. 713$721,1874$.

[4] J. F. Thorpe and W. A. Shaw, Eds., Developments in Theoretical and Applied Mechanics, vol. 3, Pergamon Press, Oxford, UK, 1967.

[5] P. S. Gupta and A. S. Gupta, "Squeezing flow between parallel plates," Wear, vol. 45, no. 2, pp. 177-185, 1977.

[6] A. F. Elkouh, "Fluid inertia effects in squeeze film between two plane annuli," Transactions of the ASME, Journal of Tribology, vol. 106, no. 2, pp. 223-227, 1984.

[7] R. L. Verma, "A numerical solution for squeezing flow between parallel channels," Wear, vol. 72, no. 1, pp. 89-95, 1981.

[8] P. Singh, V. Radhakrishnan, and K. A. Narayan, "Squeezing flow between parallel plates," Ingenieur-Archiv, vol. 60, no. 4, pp. 274281, 1990.

[9] P. J. Leider and R. B. Bird, "Squeezing flow between parallel disks. I. Theoretical analysis," Industrial and Engineering Chemistry Fundamentals, vol. 13, no. 4, pp. 336-341, 1974.

[10] G. Domairry and A. Aziz, "Approximate analysis of MHD dqueeze flow between two parallel disks with suction or injection by homotopy perturbation method," Mathematical Problems in Engineering, vol. 2009, Article ID 603916, 19 pages, 2009.

[11] S. Islam, H. Khan, I. A. Shah, and G. Zaman, "An axisymmetric squeezing fluid flow between the two infinite parallel plates in a porous medium channel," Mathematical Problems in Engineering, vol. 2011, Article ID 349803, 10 pages, 2011.

[12] I. Ullah, H. Khan, and M. T. Rahim, "Approximation of first grade MHD squeezing fluid flow with slip boundary condition using DTM and OHAM," Mathematical Problems in Engineering, vol. 2013, Article ID 816262, 9 pages, 2013.

[13] A. M. Siddiqui, S. Irum, and A. R. Ansari, "Unsteady squeezing flow of a viscous MHD fluid between parallel plates," Mathematical Modelling and Analysis, vol. 13, pp. 565-576, 2008.

[14] R. J. Grimm, "Squeezing flows of Newtonian liquid films an analysis including fluid inertia," Applied Scientific Research, vol. 32, no. 2, pp. 149-166, 1976.

[15] M. M. Rashidi, A. M. Siddiqui, and M. T. Rastegari, "Analytical solution of squeezing flow between two circular plates," International Journal for Computational Methods in Engineering Science and Mechanics, vol. 13, no. 5, pp. 342-349, 2012.

[16] H. M. Laun, M. Rady, and O. Hassager, "Analytical solutions for squeeze flow with partial wall slip," Journal of Non-Newtonian Fluid Mechanics, vol. 81, no. 1-2, pp. 1-15, 1999.
[17] S. Ishizawa, "The unsteady flow between two parallel discs with arbitrary varying gap width," Bulletin of the Japan Society of Mechanical Engineers, vol. 9, no. 35, pp. 533-550, 1966.

[18] C. le Roux, "Existence and uniqueness of the flow of secondgrade fluids with slip boundary conditions," Archive for Rational Mechanics and Analysis, vol. 148, no. 4, pp. 309-356, 1999.

[19] L. J. Rhooades, R. Resnic, T. O'Bradovich, and S. Stegman, "Abrasive flow machining of cylinder heads and its positive effects on performance and cost characteristics," Tech. Rep., Dearborn, Mich, USA, 1996.

[20] C. L. M. H. Navier, Memoirs de l'Cademie Royale des Sciences de l'Institut de France, vol. 1, Royale des Sciences de l'Institut de France, 1823.

[21] A. Ebaid, "Effects of magnetic field and wall slip conditions on the peristaltic transport of a Newtonian fluid in an asymmetric channel," Physics Letters Section A: General, Atomic and Solid State Physics, vol. 372, no. 24, pp. 4493-4499, 2008.

[22] J.-H. He, "Some asymptotic methods for strongly nonlinear equations," International Journal of Modern Physics B: Condensed Matter Physics, Statistical Physics, Applied Physics, vol. 20, no. 10, pp. 1141-1199, 2006.

[23] S. J. Liao, Proposed homotopy analysis techniques for the solution of nonlinear problems [Ph.D. thesis], Jiao Tong University, Shanghai, China, 1992.

[24] J.-H. He, "Homotopy perturbation method for solving boundary value problems," Physics Letters A, vol. 350, no. 1-2, pp. 87$88,2006$.

[25] J.-H. He, "Approximate analytical solution for seepage flow with fractional derivatives in porous media," Computer Methods in Applied Mechanics and Engineering, vol. 167, no. 1-2, pp. 57-68, 1998.

[26] V. Marinca and N. Herişanu, "Application of optimal Homotopy asymptotic method for solving nonlinear equations arising in heat transfer," International Communications in Heat and Mass Transfer, vol. 35, no. 6, pp. 710-715, 2008.

[27] V. Marinca, N. Herişanu, C. Bota, and B. Marinca, "An optimal homotopy asymptotic method applied to the steady flow of a fourth-grade fluid past a porous plate," Applied Mathematics Letters, vol. 22, no. 2, pp. 245-251, 2009.

[28] V. Marinca, N. Herişanu, and I. Nemeş, "Optimal homotopy asymptotic method with application to thin film flow," Central European Journal of Physics, vol. 6, no. 3, pp. 648-653, 2008.

[29] J. Ali, S. Islam, and G. Zaman, "The solution of multipoint boundary value problems by the optimal Homotopy asymptotic Method," Computers and Mathematics with Applications, vol. 59, no. 6, pp. 2000-2006, 2010.

[30] M. Idrees, S. Islam, and S. Haq, "Application of the optimal homotopy asymptotic method to squeezing flow," Computers and Mathematics with Applications, vol. 59, no. 12, pp. 38583866, 2010.

[31] J. Ali, S. Islam, H. Khan, and S. I. Shah, "The optimal homotopy asymptotic method for the solution of higher-order boundary value problems in finite domains," Abstract and Applied Analysis, vol. 2012, Article ID 401217, 14 pages, 2012.

[32] H. Khan, S. Islam, J. Ali, and I. Ali Shah, "Comparison of different analytic solutions to axisymmetric squeezing fluid flow between two infinite parallel plates with slip boundary conditions," Abstract and Applied Analysis, vol. 2012, Article ID 835268, 18 pages, 2012.

[33] T. C. Papanastasiou, G. C. Georgiou, and A. N. Alexandrou, Viscous Fluid Flow, Library of Congress Cataloging, CRC Press, New York, NY, USA, 1999. 


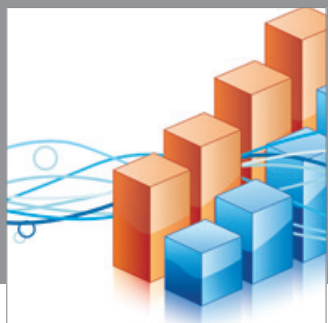

Advances in

Operations Research

mansans

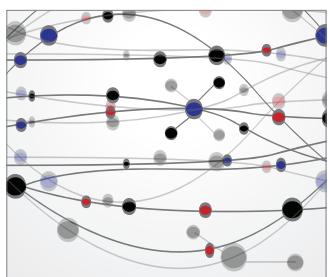

The Scientific World Journal
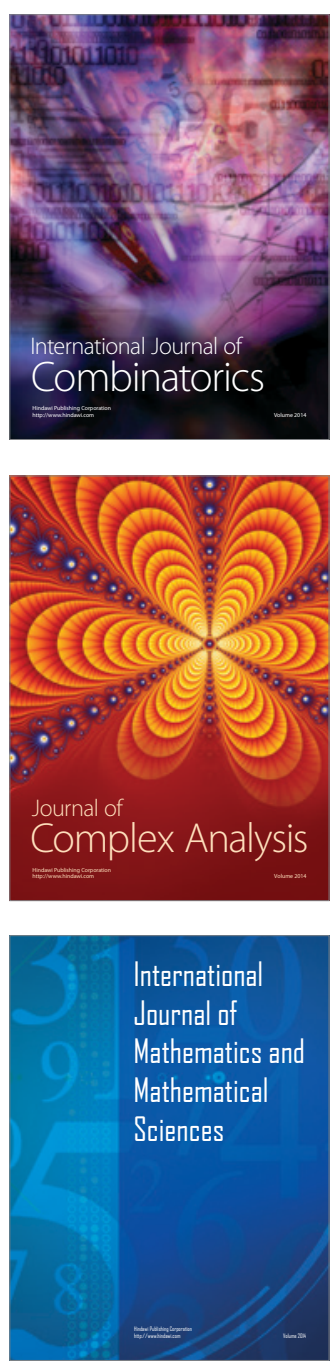
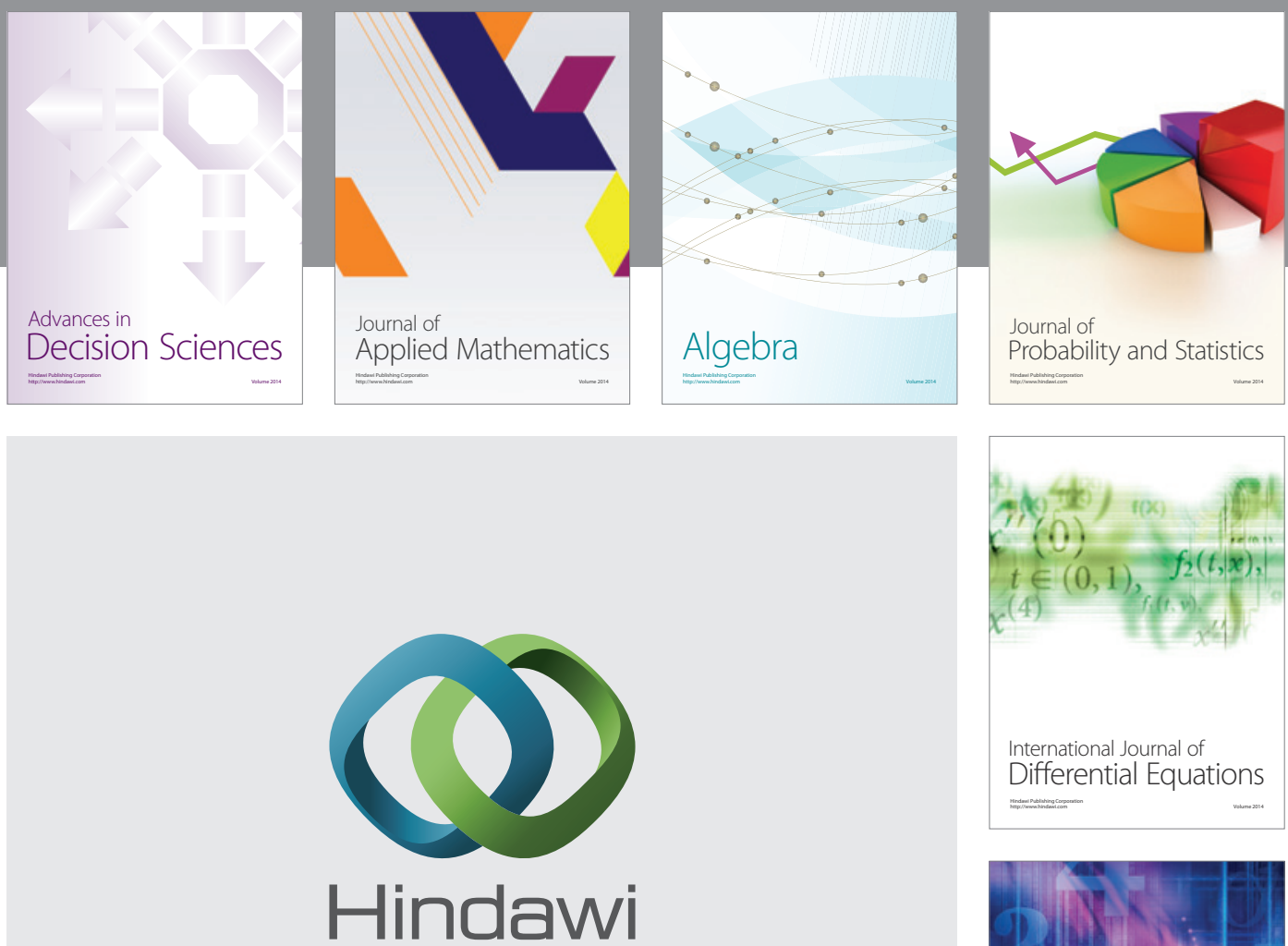

Submit your manuscripts at http://www.hindawi.com
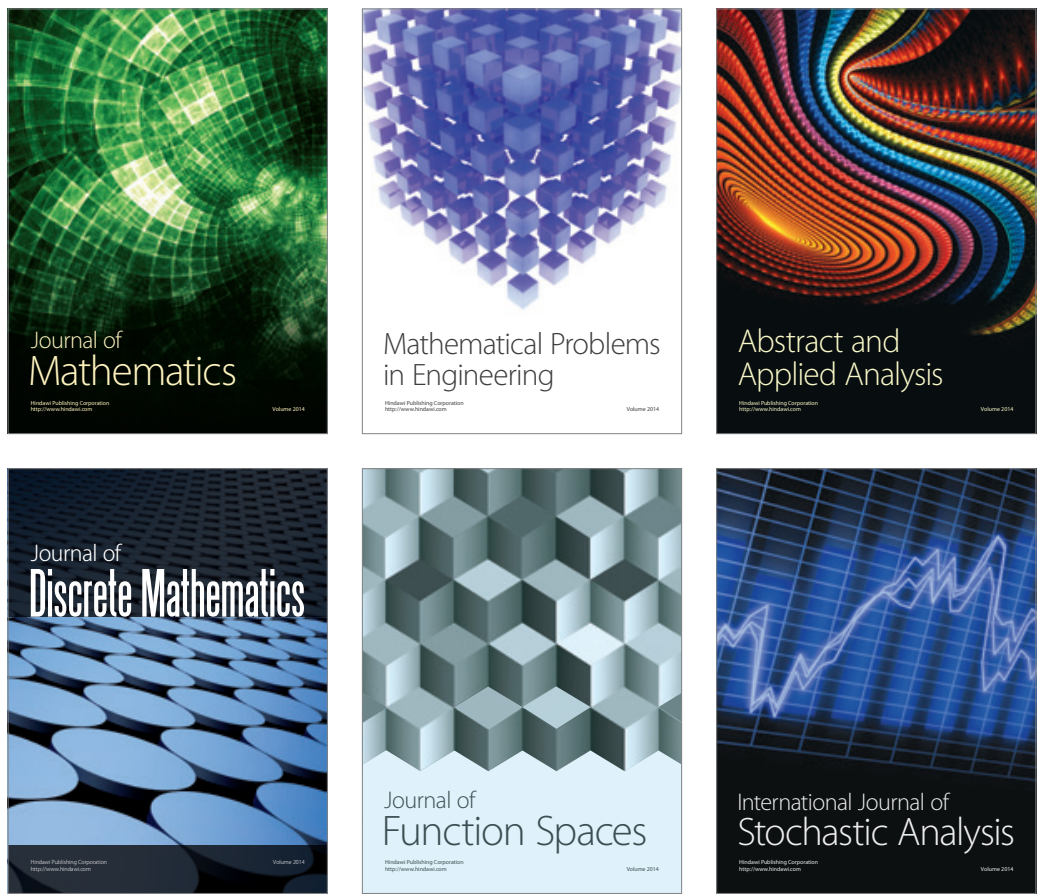

Journal of

Function Spaces

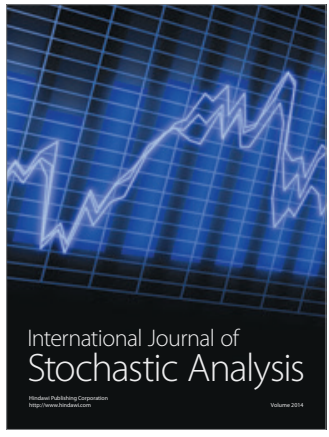

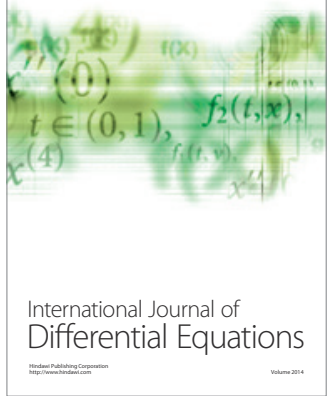
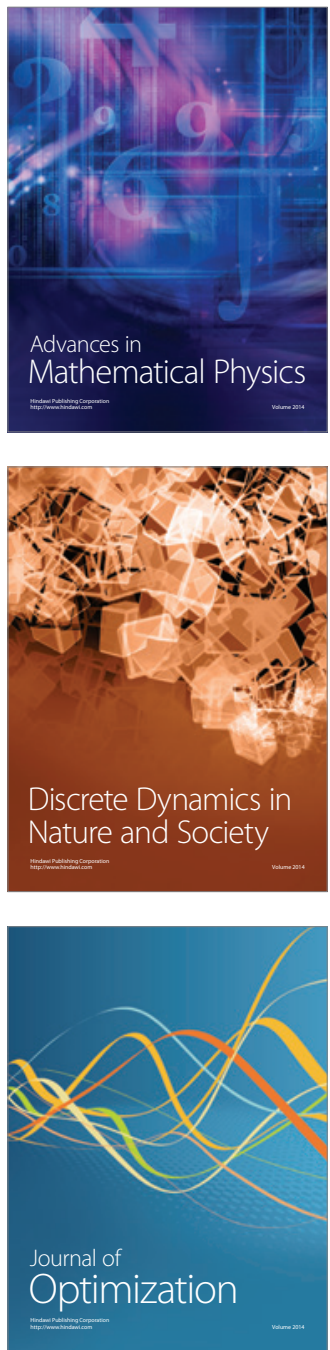